DIGITAL IMAGING BASICS

\title{
Properties of Digital Images in Radiology
}

\author{
John A. Correia and Nathaniel M. Alpert
}

$\mathbf{D}^{\prime}$ URING the past 15 years the use of digitized images in radiology has proliferated. It is reasonable to expect that within a few years virtually all radiologic images will be available in digital form and further, that this will be the predominant mode of image presentation within radiology. Historically, nuclear medical images were the first to be regularly digitized because they have relatively low resolution and contrast compared to other medical images. ${ }^{2}$ The computer hardware available 15 years ago was so limited in both speed and capacity that only these low-information-content images could be efficiently handled in the digital regime.

$\mathrm{X}$-ray computed tomography (CT) of the early $1970 \mathrm{~s},{ }^{2}$ a modality that is intrinsically digital in the sense that the output is a computed image, was the first modality to require a computer for image production and it naturally followed that the digital images produced would be displayed electronically. This modality has set the stage for the digital imaging revolution that is taking place today. For the first time, radiologists were exposed to the advantages of postacquisition image manipulation and began to accept the usefulness of such capability.

Since the introduction of CT, there have been several developments in digital imaging including computer-dependent modalities such as magnetic resonance imaging (MRI) $)^{3,4}$ and the application of digital image acquisition and display methods to intrinsically analog modalities such as planar radiography and fluoroscopy ${ }^{6}$ (called digital radiography or DR and sometimes digital fluoroscopy or DF) and real-time ultrasound (US).$^{7,8}$ Further, the availability of digital images in the various modalities has led to the development of computationally-derived images which represent physiological quantities. ${ }^{9,10}$

From the Department of Radiology, Massachusetts General Hospital, Boston.

Address reprint requests to John A. Correia, PhD, Department of Radiology, Massachusetts General Hospital, 32 Fruit St, Boston MA 02114.

() 1988 by W.B. Saunders Company.

0897-1889/88/0101-0006\$03.00/0
The major advantages of digital imaging over conventional film-based imaging are higher efficiency detection systems may be used thus limiting patient radiation dose, the possibility for post-acquisition-processing data exists, electronic displays, which have a high degree of flexibility, can be used, and digital images may be archived and retrieved efficiently from digital data bases.

Because of the present and future impact of digital imaging, it is essential that the radiologist become familiar with digital image production, storage, and manipulation to most effectively practice his/her profession. It is the purpose of this article to address one aspect of this body of knowledge, namely the basic properties of digital images. The following sections include discussions of a number of these properties including digital image production, size, contrast, and storage.

\section{PROPERTIES OF A DIGITAL IMAGE}

A digital image can be thought of as a collection of digital storage elements such that there is a one-to-one correspondence between a location in the image field and a unit of digital storage. For example, the field encompassed by a plane film may be divided into discrete elements and the average value of $x$-ray absorption in each element assigned a unique digital location, or the value at a reconstructed CT image element may be assigned a similar unique location. In the case of a projection image such as a plane film, the element is called a pixel (a contraction of picture element) and in the case of a cross-sectional,volumetric modality such as CT it is called a voxel (volume element). It is convenient to think of such a digital image as a matrix having an $\mathrm{x}$ - and $y$-coordinate which uniquely specify the location of a given element (Fig 1). The number that specifices this location is called a "digital address" within a computer memory or mass storage device.

The actual information within a computer memory or storage medium need not be organized in exactly this way. Typically, the digital image can be thought of as an $N$ by $M$ matrix 


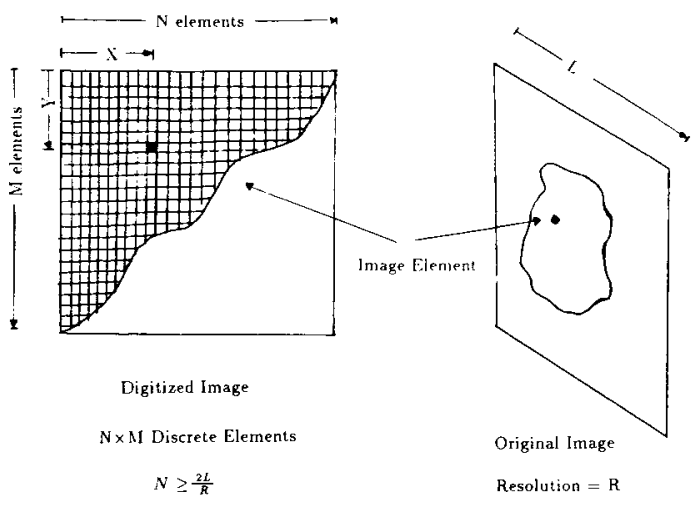

Fig 1. Digital image matrix format showing $x$ - and $y$-coordinates and one-to-one correspondence between image field location and digital image element.

having $\mathbf{N} \times \mathbf{M}$ discrete elements. For computational convenience, $\mathbf{N}=\mathbf{M}=2^{1}$ where 1 is in the range of five to 11 . This gives rise to matrices having from $1024(32 \times 32)$ to $4,194,304$ $(2048 \times 2048)$ elements. The digital image size is related to the spatial resolution represented in a given image.

Each element in a digital image is typically a byte ( 8 bits), a word (16 bits), or a long word ( 32 bits) representing, in integer format, the value of the image parameter associated with that image. In general an $\mathrm{n}$ bit integer can represent a range of numbers from 0 to $2^{\mathrm{n}}-1$. Thus a byte can represent a range from 0 to 255 while a word can represent a range from 0 to 65,536 as illustrated in Fig 2. This parameter is often called the "image depth" and is related to the fineness of the contrast differences that can be represented.

\section{CHOICE OF DIGITAL IMAGE SIZE}

The minimum number of elements needed to represent a digital image from a given modality depends on the intrinsic spatial resolution of that modality. This relationship arises from the need to have sufficient elements to represent the image without degradating its intrinsic resolu-

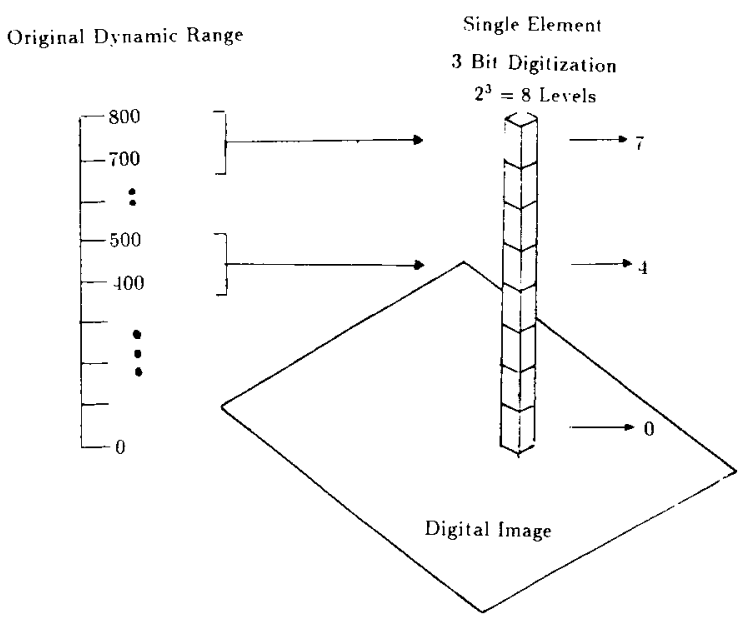

Fig 2. Digitization of contrast in each image element.

tion and is dictated by a fundamental result of signal processing theory called the sampling theorem. ${ }^{11}$ The sampling theorem requires that for a given spatial resolution, $R$, defined by the physical limitations of the modality, it is necessary to have at least two image elements per resolution length to preserve the information in a digitized image. In practice, more than this number of elements is needed. For example, if a given image has a characteristic dimension 1 and a resolution $R$ then a matrix with a dimension of at least $21 / R$ is required. In the case of a nuclear medicine device with resolution of $8 \mathrm{~mm}$ imaging a 300 $\mathrm{mm}$ object, one would require a matrix with at least 94 elements on a side, leading to a probable choice of a $128^{2}$ matrix. Characteristic digital matrix sizes for other modalities for a $300 \mathrm{~mm}$ object are shown in Table 1.

If a digital image does not meet the minimum spatial sampling requirement imposed by the sampling theorem, then information is lost in the digitization process. This may result in a phenomenon called aliasing in which artifacts or distortions are introduced into the image. An example of this in every day life is the appear-

Table 1. Typical Digital Image Matrix Sizes for $300 \mathrm{~mm}$ Diameter Object in Various Imaging Modalities

\begin{tabular}{lcccc}
\hline \multicolumn{1}{c}{ Modality } & Spatial Resolution $(\mathrm{mm})$ & $\begin{array}{c}\text { Number of Samples } \\
(2 \mathrm{D} / \mathrm{r})\end{array}$ & Minimum Matrix & Commonly Used Matrix \\
\hline Nuclear medicine & 8.0 & 75 & $75^{2}$ & $128^{2}$ \\
X-ray CT & 2.0 & 300 & $320^{2}$ & $512^{2}$ \\
MRI & 2.0 & 300 & $300^{2}$ & $256^{2}$ \\
DR & 0.4 & 1500 & $2048^{2}$ & $512^{2}$ \\
UItrasound & 3.0 & 200 & $200^{2}$ & $256^{2}$ \\
\hline
\end{tabular}




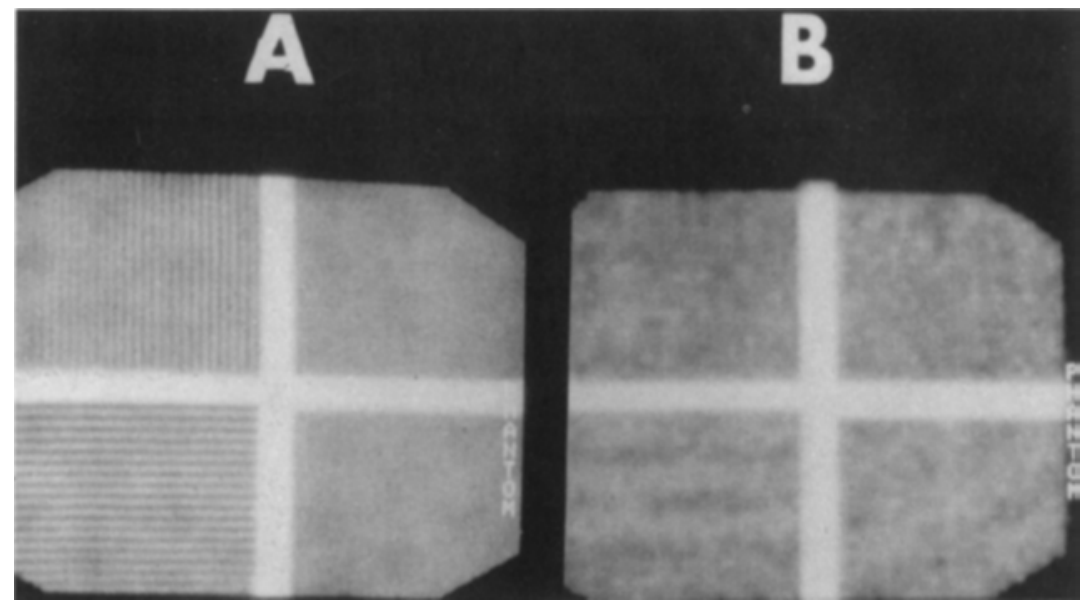

Fig 3. Example of aliasing. Bar phantom acquired on a Gamma Camera using a $512^{2}$ matrix (A) and a $128^{2}$ matrix (B). The higher resolution representation of the bars on the lower left is accurate while the lower resolution image shows an artifact consisting of thicker bars.

ance in a television image of a superimposed pattern when an object containing parallel lines with spacing finer than the television's discrete vertical sampling of 525 lines is viewed. In medicine, these artifacts may manifest themselves as unwanted or distorted structures in the image as illustrated in Fig 3.

In practice, it is sensible to choose a matrix size that is sufficient to represent the given image and not a larger one. The larger a matrix, the more mass storage it requires and the longer it takes to store, recover, transmit, and manipulate it.

One of the factors that has limited, and perhaps still limits the digitization of some images, particularly very high resolution images such as planar radiographs, is that the storage required to digitize such images at full resolution is very large. For example, to preserve all the resolution in a chest film having $0.2 \mathrm{~mm}$ intrinsic resolution requires a matrix image size of $4096 .^{2}$ Until recently, the cost of storage and low transfer speed of matrices of this size within the computer and storage media have prohibited their use. However, a matrix size large enough to maintain the full resolution is not always necessary since the information content of the measured data rather than the resolution may be the limiting factor. Factors such as image contrast and structure size may contribute to defining information content.

\section{CHOICE OF IMAGE DEPTH}

The contrast resolution of an image is qualitatively defined as the fineness with which differences in the imaged parameter can be detected for a given image at a given resolution. An image may be described by the number of elements and the number of bits of contrast needed, for example $512 \times 512 \times 12$ bits deep (also $512^{2}$ by 12 bits).

For images of parameters measured directly from ionizing radiation, the contrast resolution is limited by the number of photons detected. Since the detected photons follow Poisson statistics, contrast resolution is a percentage of the dynamic range of the values measured ( 0 to $N$ ) given by $\sqrt{n / N}$, where $n$ is the average number of events recorded in a given image element. For CT images, there is some amplification of the basic Poisson noise due to the reconstruction process, but the relative behavior of the signal to noise ratio is similar to that for directly acquired images. In modalities such as MRI and US, the signal to noise ratio of the image elements depends on the amount of fluctuation in the measured signals and can be improved by the process of signal averaging in which the signal is measured multiple times and averaged. Typical contrast resolutions for the radiologic imaging modalities are given in Table 2.

As is the case with spatial sampling, the undersampling of the contrast range of an image can cause artifacts. The most typical artifact is the appearance of false edges or contours in the image. These can be confused with true edges and structures resulting in errors in clinical findings. An example of such false contours is shown in Fig 4.

Nuclear medical images, for example, have an intrinsic contrast of about $5 \%$ or a signal to noise ratio of 20 due to the limited number of photons 
Table 2. Typical Contrast Resolution and Digital Image Depth for Medical Imaging Modalities

\begin{tabular}{|c|c|c|c|c|}
\hline Modality & Intrinsic Contrast (\%) & Minimum No. of Bits & $\%$ Grey Levels & Usual No. of Grey Levels \\
\hline Nuclear medicine/pet & $3.0=10$ & $5(6)$ & 32 & 64 \\
\hline $\mathrm{X}$-ray $\mathrm{CT}$ & $0.5=1.0$ & $8(16)$ & 256 & 4096 \\
\hline MRI & $1.0=3.0$ & $8(16)$ & 256 & 4096 \\
\hline DR & 0.2 & $10(8)$ & 1024 & 256-1024 \\
\hline Ultrasound & $1.0=5.0$ & $8(4)$ & 256 & 256 \\
\hline
\end{tabular}

detected (typically about 500,000 events per image). This means that differences in contrast or radioactive density of $5 \%$ are detectable in adjacent image elements and smaller differences are not statistically significant. Since the same sampling theorem considerations apply to contrast, at least 40 discrete numbers are required to represent the information in an image element. In practice, a byte representing a range of 0 to 255 may be used if the data are normalized to that range. A CT image, on the other hand has a contrast resolution of $0.2 \%$ requiring at least

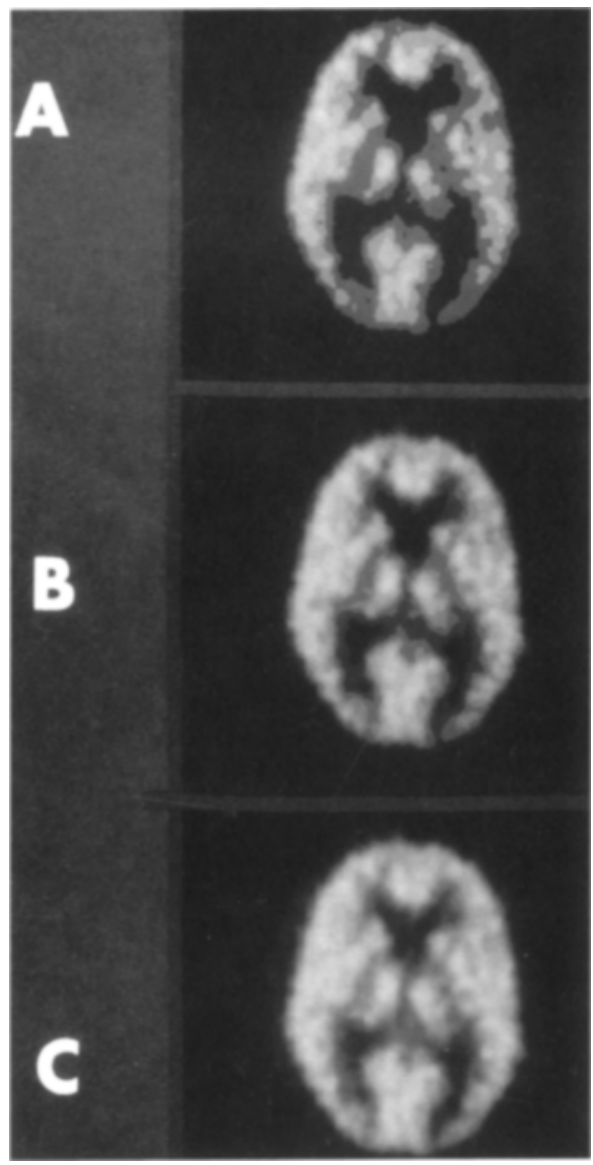

Fig 4. PET brain image displayed with 64 (A), 16 (B) and 8 (C) grey levels. Artificial contours may be seen in the images with coarser grey scales.
1,000 discrete numbers. A word representing a range of 0 to 65,536 (actually $\pm 32,678$ ), the next convenient power of two, is used. A digital radiography image has similar contrast resolution.

\section{DIGITIZATION AND TEMPORAL RESOLUTION}

The process by which digital images are produced usually involves the conversion of an analog electrical signal such as a voltage, a current, or a television signal to digital spatial and contrast information. This transformation is achieved by an electronic device called an analog to digital converter (ADC). In devices such as scintillation cameras which have low event detection rates and $\mathrm{x}$-ray $\mathrm{CT}$ scanners where a large number of individual detectors are served by multiple ADCs which digitize detector current, limitations on the digital conversion process are minor and digitized matrix sizes and depths appropriate to the information in the original data can be achieved in most instances. MRI also falls into this category since at any one time, data associated with only one line making up the image are being digitized.

In modalities such as digital fluoroscopy, where a television signal representing a whole image field is to be converted to a digital image, the properties of the resulting digital image are limited by the digitization rate of the ADC. The intrinsic television frame rate is 30 frames per second, the contrast of the fluoroscopic image would support a contrast range requiring up to 12 bits; the spatial resolution of the fluoroscopic image would support digital matrix sizes of up to $2,048^{2}$; and the fastest ADCs commonly available today can digitize at a rate of about 10 million events per second. The combination of potentially large matrices, image depths, and potentially high frame rates requires that some tradeoff be made in digitizing these images. If an ADC which digitizes at a maximum rate of 10 Mbytes/sec is used, the required digitization rate to collect 30 frames per second of matrix size $512^{2}$ and depth 12 bits would be approximately 
12 Mbytes per second, beyond the capacity of the ADC. The options available to overcome this problem are: (1) to give up spatial resolution by decreasing matrix size; (2) to give up contrast resolution by reducing depth; or (3) to give up temporal resolution by decreasing the number of frames per second collected. If high resolution television technology having 1,024 or 2,048 lines per screen is used, the problem becomes even more acute. The size of the field can be reduced by "coning down" the x-ray beam to preserve spatial resolution over a smaller field of interest at high frame rates.

Another limitation of the digital image collection rate is the time required to transfer images internally in the computer, ie, the transfer of images along the computer communications bus and write times to mass storage disks. This problem can be moderated by designing special image acquisition memories that hold a large number of images coupled with special circuits which store data from the ADCs directly into the memory. Computer memory is relatively inexpensive today and storage systems containing 100 Mbytes are commonly available. Such a memory would hold ten of the digital fluoroscopy images described above. Having two independent memories and appropriate computer hardware would allow the uninterrupted collection of images at high rates by transferring data from one memory to mass storage while data is being acquired into the other. This technique is called double buffering, or when extended to multiple memories, multi-buffering.

\section{STORAGE OF DIGITAL IMAGES}

The overall storage required for a digital image of various modalities varies considerably as illustrated in Fig 5. The two parameters defining the digital image size, the spatial extent and the contrast depth, may be chosen to maximize storage capability. For example, dynamically acquired nuclear medical images, in which the intrinsic contrast resolution is poor due to statistical limitations, may be stored as $64^{2}$ images having a bit depth of only 8 bits. While digital fluoroscopy images, which are typically limited by the speed of the ADCs which digitize television signals, are typically $512^{2}$ as limited by the vertical resolution of the television camera and have signal to noise ratios which may require up to 12 bits of contrast resolution. Newer televi-

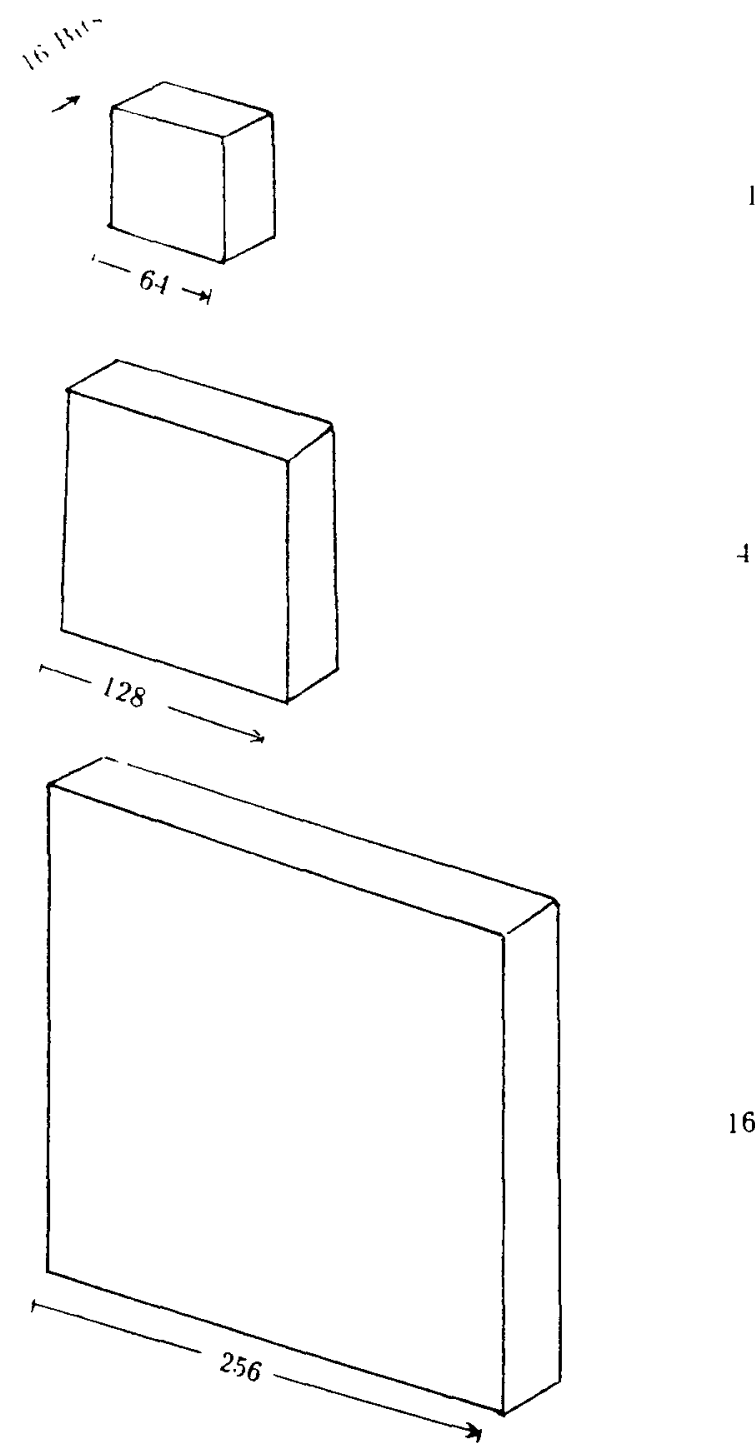

Fig 5. Schematic representation of relative storage requirements for digital images of different sizes.

sion technologies and direct digital detection systems will require image matrix sizes of $1,024^{2}$ or even $2,048^{2}$ to take full advantage of available resolution. Other modalities fall in between these extremes (Fig 5).

\section{CONCLUSION}

The use of digital images in radiology has become widespread and will likely become universal within radiology departments in the near future. Therefore, radiologists must be conversant with properties of digital images including spatial and contrast resolution and their implications in terms of their requirements in viewing and interpreting such images. 


\section{REFERENCES}

1. Metz CE, Pizer SM, Brownell GL, (eds): Information Processing in Scintigraphy. United States Energy Research and Development Agency, Conference 730687, 1973

2. Hounsfield GN: Computerized transverse axial scanning: Description of the system. Br J Radiol 46:1016-1022, 1973

3. Lauterbur PC: Image formation by induced local interactions: Examples employing nuclear magnetic resonance. Nature, 242:190-191, 1973

4. Hinshaw WS, Andrew ER, Bottomley PA, et al: Display of cross-sectional anatomy by nuclera magnetic resonance imaging. Br J Radiol 51:273-280, 1978

5. Drew PG: The coming revolution in digital radiography. Diagn Imaging, 3:10-1 1, 1982
6. Sackett JF, Crummy AB, Strother CM, et al: The computerized flouroscopy: Digital subtraction for IV angiography and arteriography. AJR 135:1131-1140, 1980

7. Wells PN: Biomedical Ultrasonics, San Diego, Academic, 1977

8. Wells PN: Real time scanning systems. Clin Diagn Ultrasound, 5:69-73, 1980

9. Phelps ME, Mazziotta JM, Huang SC: Study of cerebral function with positron computed tomography. J Cereb Blood Flow Metab, 2:113-162, 1982

10. Cahn $J$ and Lassen NA (eds): New brain imaging techniques in cerebrovascular disease. Libbey Eurotext, 1985

11. Barrett HA and Swindell W: Radiologic Imaging, Volume I. San Diego, Academic, 1981 